\title{
ACTIVE ADAPTIVE ARRAYS: THE ASTRON APPROACH TO SKA
}

\author{
RICHARD G. STROM ${ }^{1,2}$, BART SMOLDERS $^{1}$ and ARNOLD VAN ARDENNE ${ }^{1}$ \\ ${ }^{1}$ ASTRON, P.O. BOX 2, 7990 AA Dwingeloo, Netherlands; \\ ${ }^{2}$ Astronomical Institute 'Anton Pannekoek', University of Amsterdam, Netherlands
}

\begin{abstract}
It has been argued, for a number of reasons, that the next generation radio telescope should be a multi-element interferometer with a collecting area of about $\mathrm{I} \mathrm{km}^{2}$. The remaining parameters of such an instrument - frequency range, angular resolution, instantaneous bandwidth, etc. - will be science driven. The requirements for propagation studies are briefly discussed, and it is pointed out how variable-source confusion may differ from the normal variety. Finally, the Dutch project to achieve a large collecting area using adaptive arrays of active antennas is described. A systematic approach has been adopted, with the construction of arrays of increasing complexity to test design features at each state. Recently, development of a low frequency array (LOFAR) has become an additional option. It would facilitate tests of some of the larger instrument's features, and provide real data on the inlluence of the ionosphere and interfering sources.
\end{abstract}

\section{Introduction}

Most telescopes, radio or otherwise, seek to maximize the signal from the object of interest, while reducing background noise to the minimum possible. In many radio frequency bands, the noise produced by the receiver can be made lower than natural sources such as the sky background or the atmosphere. It has therefore been argued (see, in particular, the contributions by Braun, Taylor, Briggs, and Giovanelli, in Strom et al., 1996) that the next generation radio telescope will necessitate a large increase in collecting area compared with today's instruments: specifically, a total collecting area of $1 \mathrm{~km}^{2}$. Hence the project's designation as the Square Kilometer Array (SKA).

How can we achieve the huge SKA collecting area for a reasonable price?* Various approaches have been suggested (most have actually been used at some time or other for smaller instruments), and they all have to solve the technical problem of intercepting the broadest possible wavefront and transferring its signal in a (nearly) lossless manner to electronic circuitry. The solutions being investigated by various groups range from a small number (30-50) of large monolithic reflectors (the KARST project in China; the Canadian LAR), through a great many (100 s to $1000 \mathrm{~s}$ ) smaller steerable paraboloids (the SETI Institute's 1HT; research in India), to arrays of many $1000 \mathrm{~s}$ of dipoles, utilizing planar reflectors (as we foresee), or

* 'Reasonable' has been taken to mean of order $\$ 500 \mathrm{M}$, or about $\$ 500 \mathrm{~m}^{-2}$ including not just the reflecting surface, but that and everything else from electronics to infrastructure and software. 
lens-type collectors (Australian investigations of the Luneberg lens). Some of the other projects are reported elsewhere in these proceedings (see also Van Ardenne, 1999).

\section{SKA and Propagation Studies: Instrumental Desiderata}

Even having set the collecting area (and hence, to some extent, the sensitivity), there are still other instrumental parameters to be matched to the scientific goals we astronomers have in mind. Given the topic of this Colloquium, let us consider how some of the other parameters can be best matched to studies of propagation. In particular, we still have to decide upon the frequency range, the instantaneous bandwidth, number of frequency channels, angular resolution and number of beams (among others).

Although the sensitivity may have been set by the $1 \mathrm{~km}^{2}$ size, it is worth keeping in mind that for rapidly varying sources (pulsars, low frequency IPS, etc.), the sensitivity and shortest timescale to be probed determine the amplitude $(\Delta S)$ of the weakest changes which can be studied $\left(\Delta S>S_{\text {noise }} /(\Delta \nu \Delta \tau)^{1 / 2}\right)$. The frequency structure of the type of variability being investigated will also affect the sensitivity by setting the largest usable bandwidth.

Propagation studies are carried out on radio sources ranging from pulsars (mainly at low frequencies) to compact quasars, AGNs, and the transient radio emission from gamma-rays bursts. To cover all of these types of objects would require a frequency range of roughly $50 \mathrm{MHz}$ to $10 \mathrm{GHz}$. A bandwidth of $10 \mathrm{~s}$ of $\mathrm{MHz}$ at the lower frequencies, perhaps $100 \mathrm{MHz}$ at the higher ones, and 20-100 frequency channels, would be desirable.

As for the angular resolution required, the problem has two aspects: the resolution needed to study scatter broadening, and the minimum to prevent source confusion, given the instrument's sensitivity. To study source scatter broadening will usually require VLBI baselines (angular scales of $10 \mathrm{~s}$ of m.a.s. or less), although highly scattered sources in the direction of the Galactic Center can be broadened by $1^{\prime \prime}$ arc or more at $18 \mathrm{~cm}$ (Frail et al., 1994), with the angle increasing as, $\theta \propto \lambda^{2}$. This means in any event an angular resolution of $1^{\prime \prime}$ arc or better, implying baselines which extend to several hundred $\mathrm{km}$.

Similar resolutions are required for SKA when used for continuum imaging, if confusion is not to dominate the total noise budget. When it comes to studies (rapidly) varying sources, the confusion we have to worry about is that of having another variable source in the instrumental beam. Since some fraction of all sources is too large to vary (intrinsically or extrinsically), the problem of variablesource confusion should be less severe than the normal variety. However, as weaker sources are on average more compact, a larger proportion of them will scintillate. Moreover, in the case of shortterm variability, we have to consider the confusion 
level of the instantaneous ('snapshot') beam rather than that produced by the better $u, v$-coverage of long tracks.

The possibility of multi-beaming would be useful for certain types of propagation studies. For shortterm variations produced in a nearby scattering screen, one could simultaneously observe several compact AGNs and pulsars to probe the structure and extent of the scatterer. Multiple beams might also be used to facilitate multifrequency observing.

\section{SKA Realization with Arrays: The ASTRON Approach}

When considering how we might best approach the problem of achieving a large collecting area with sensitive electronics for a reasonable price, several local factors played a role. Holland is a flat, fairly windy country with a temperate climate, though in winter we can have freezing rain and snow. ASTRON has considerable experience in the areas of interferometry, receivers, and large correlators; but much less in-house expertise when it comes to large mechanical structures. Furthermore, the price of electronic components continues to decline (Moore's Law), while that of large structures (steel) will probably change little. Hence, early on, the following guidelines were enunciated:

- Keep it flat

- No moving parts

- Use Si, not Fe

The resulting project to develop active adaptive arrays (Van Ardenne et al., 2000) has moved stepwise from single elements to a small (16-element) array with a single beam, and now to a 1024-element, 32-beam antenna. The prototype of this THousand Element Array (THEA), which operates from $0.5-1.7 \mathrm{GHz}$ with a 20 $\mathrm{MHz}$ IF, will be tested with sky sources starting this summer. One of the features of the adaptive array is the ability not only to direct each beam to an arbitrary location on the sky, but to also tailor the beam shape to minimize its response to unwanted signals (interference suppression). This occurs dynamically, so that observations can be made in a changing interference environment, and tests have demonstrated the viability of the concept.

We are now four years into a ten-year project which will culminate in a prototype array, a 100-m class instrument usable for radio astronomy in its own right, but also potentially one element of the SKA. A new branch to this effort has recently opened up, a low-frequency array (LOFAR) which might operate over the $\simeq 10-100 \mathrm{MHz}$ range. Scientifically, LOFAR would produce a survey of the whole sky visible to it, as well as being able to map and monitor a variety of sources at low frequencies. Instrumentally, it provides us with a testbed for investigating some of the problems we are likely to encounter in SKA. External interference and ionospheric effects are two of particular importance. The LOFAR effort can thus be seen as parallel and complementary to ASTRON's SKA development. 
Finally, what about the costs? The development thus far has seen the price per element drop by nearly two orders of magnitude, to several thousand \$US for each THEA element. For SKA to be affordable, we need to bring the price down to a few $\$$ per element. This goal is still within our sights, but there's quite a way to go yet!

\section{Acknowledgements}

RGS is grateful to the LOC of IAU Colloquium 182 for providing partial financial support. ASTRON (The Netherlands Foundation for Research in Astronomy) receives financing from the Netherlands Organisation for Scientific Research (NWO).

\section{References}

Van Ardenne, A.: 1999, The technology challenge for the next generation radio telescopes, in: A.B. Smolders and M.P. van Haarlem (eds.), Perspectives on Radio Astronomy: Technologies for Large Antenna Arrays, ix-xviii, Astron, Dwingeloo.

Van Ardenne, A., Smolders, B. and Hampson, G.: 2000, Active adaptive antennas for radio astronomy; results of the R\&D program toward the Square Kilometer Array, Proc. SPIE Conf. $\mathbf{4 0 1 5}$ Radio Telescopes, H.R. Butcher (ed.), 420-433.

Frail, D.A., Diamond, P.J., Cordes, J.M. and van Langevelde, H.J.: 1994, Anisotropic scattering of $\mathrm{OH} / \mathrm{IR}$ stars toward the Galactic center, Astrophys. J. 427, L43-L46.

Strom, R.G., Peng, B. and Nan, R.: 1996, Proc: 3rd Meeting of the Large Telescope Working Group \& Workshop on Spherical Radio Telescopes, International Academic Publishers, Beijing. 